\title{
O HOLISMO APLICADO AO CONHECIMENTO AMBIENTAL
}

\author{
Julio C. Wasserman ${ }^{1}$ \\ Albano R. Alves ${ }^{2}$
}

\begin{abstract}
Resumo: A abordagem holística pode ser definida de maneira aproximada como aquela que considera as infinitas interações entre os diversos componentes de um sistema complexo. Alguns autores têm chamado esta visão do funcionamento das coisas do Universo como abordagem sistêmica. As áreas em que esta abordagem pode ser aplicada variam desde a astrofísica até a gestão de negócios, mas uma área onde o holismo é particularmente útil é a ciência da natureza. A dificuldade na aplicação da abordagem holística às ciências naturais vem da inexistência de metodologias bem estabelecidas e testadas, levando os cientistas a se depararem com um imenso abismo de conhecimentos, quando são levados a entenderem processos de áreas diversas às quais está afeito. Um exemplo crítico pode ser obtido da previsão de impactos ambientais, onde a aplicação de modelos numéricos demanda uma pesada carga de conhecimento matemático, de forma que os processos sejam corretamente simulados. No caso, cada passo do processo precisa ser equacionado da maneira mais precisa possível, caso contrário, erros pequenos podem se multiplicar e gerar informações totalmente erradas. Já se observam equipes interdisciplinares sendo formadas e gerando enormes avanços no conhecimento dos processos ambientais, mostrando que, embora a formação científica seja ainda extremamente cartesiana, aprendese na prática como fazer ciência holística.
\end{abstract}

Palavras-chaves: Holismo, Meio Ambiente, Interdisciplinaridade, Modelagem numérica

Abstract: The holistic approach can be roughly defined as a philosophy that considers the endless interactions between various compartments of a complex system. Some authors have been calling this view of the mechanisms and processes that control the phenomena of the Universe as systemic approach. The areas where this approach can be applied range from astrophysics to business management, but an area where holism is particularly useful is the science of the nature. The difficulties of the application of the holistic approach to natural science, arise from the absence of well established and tested methodologies, taking the scientists to face a huge gap of knowledge as they are forced to understand processes in areas that are too far from those he is used to. A critical example can be obtained from the prevision of environmental impacts, where the application of numeric modeling requires a heavy load of mathematical knowledge, in order the processes are correctly simulated. In this case, each step of the process needs to be equationed in a very precise manner; otherwise, small errors can be magnified, generating completely false informations. Interdisciplinary teams are already being gathered to solve specific environmental problems, which yield enormous advancements on the knowledge of the environmental processes, showing that, although the scientific formation is still Cartesian, holistic science can be learned in the practice.

Key-words: Holism, Environment, Interdisciplinarity, Numeric modeling

\footnotetext{
${ }^{1}$ Programa de Pós-Graduação em Ciência Ambiental - Universidade Federal Fluminense. Av. Litorânea s/nº, Boa Viagem, Niterói, RJ, 24210-340, Brasil, e-mail: geowass@vm.uff.br

${ }^{2}$ Diretoria de Hidrografia e Navegação, Marinha do Brasil. Ponta da Armação, Niterói, 24040-010, RJ, Brasil, e-mail: albanoribeiro@terra.com.br
} 


\section{1- O HOLISMO NA CIÊNCIA AMBIENTAL}

O conceito de Ciências Naturais é muito antigo e refere-se às diversas ciências que descrevem os processos e os elementos da natureza. Esta definição extremamente ampla foi utilizada até o final dos anos 50 no Brasil, como marco de formação dos cientistas que de alguma forma queriam estudar as coisas da natureza. A biologia, a química e até a física eram matérias constituintes deste tipo de formação, abrangendo também a geologia, a geografia e a oceanografia entre outras. Esta formação generalista era possível, pois primeiramente o montante de conhecimento acumulado ainda não era tão significativo, mas, sobretudo a formação em ciências naturais não era uma demanda de sociedade, que pouco valorizava o conhecimento natural e os cientistas ainda eram considerados apaixonados e sonhadores. Com o processo de conscientização ecológica que surge em todo o mundo nas décadas de 60 e 70, que culminou com a Conferência de Estocolmo de 1972, ocorre uma "profissionalização" dos cientistas que se voltavam para o conhecimento da natureza, passando a especializar-se cada vez mais. A especialização é um processo que se observa em todas as áreas e, particularmente surtiu excelentes resultados na mecanização dos meios de produção.

Passamos então a contar com especialistas que tratavam de temas muito específicos e, apesar de interessantes, em nada contribuíam à preservação e ao equilíbrio do meio ambiente e à evolução da humanidade. A Ecologia, uma ciência que tratava das relações entre os organismos é que passa a valorizar a interação entre os componentes de sistemas. Observou-se que o funcionamento dos sistemas complexos, tais como os ecossistemas, não podia ser tratado segundo uma abordagem reducionista onde o todo é a simples soma das partes. Em um ecossistema, se um organismo interage com o outro, como seria possível entender seus respectivos comportamentos estudando-os separadamente?

O reducionismo, que é uma filosofia científica que impregna o pensamento da humanidade desde o conceito do átomo de Demócrito, no século 5 A.C., precisava ser revisto. Por volta de 1920, Jan Christiaan Smuts concebe o termo holismo com a seguinte definição: "The tendency in nature to form wholes, that are greater than the sum of the parts, through creative evolution". Segundo este conceito, a soma das partes não explica o todo. Devido à dificuldade de se explicar os processos que levam ao holismo, o conceito foi associado processos sobrenaturais. Contudo, nos últimos anos, a idéia de que nem tudo o que não entendemos é sobrenatural, vem ganhando espaço e consolidando o holismo como filosofia científica.

Apesar dos marcos teóricos, na prática ainda formamos nossos cidadãos em uma estrutura extremamente reducionista. Mesmo o Construtivismo de Piaget $^{3}$ que hoje já é aplicado nas estruturas curriculares de muitas escolas, ainda não atinge os níveis superiores da formação e cientistas ainda são formados segundo princípios educacionais antiquados. $\mathrm{O}$ holismo só aflora na formação do cientista quando este se depara com problemas que demandam interação de conhecimentos, levando-o a um traumático processo de tentativa e erro. O trabalho de Vallega (1999) é um interessante exemplo da aplicação do holismo a problemas de gestão ambiental. Outros exemplos são apresentados a seguir.

\section{2- A DISTORÇÃO UNIDISCIPLINAR: O CASO DA LAGUNA DE PIRATININGA}

Problemas ambientais são multifacetados e imbricados, sendo que todos os aspectos, sejam físicos, biológicos, químicos, sociais, etc. devem ser tratados de maneira integrada. Contudo,

\footnotetext{
${ }^{3}$ Evidentemente, o Construtivismo sozinho não é capaz de formar cidadãos holísticos, mas é um passo no sentido de levar o cidadão a construir um conhecimento interdisciplinar.
} 
muitos técnicos ainda pensam em problemas

reservatório e saída”.

ambientais como sistemas simples de "entrada,

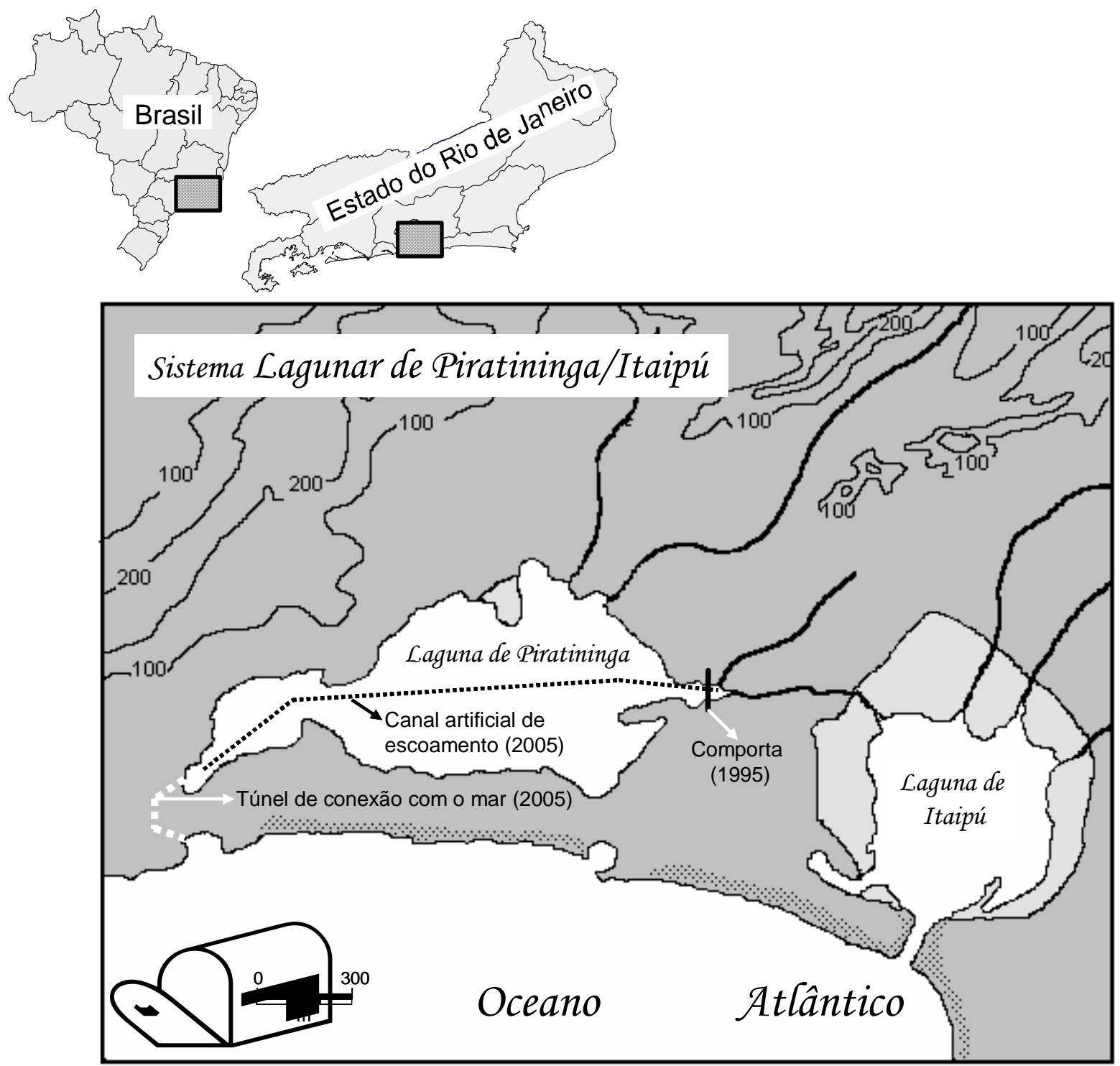

Figura 1: Laguna de Piratininga com indicação das obras realizadas em 1995 e projetadas para 2005.

Um exemplo da falta de visão do todo na aplicação de soluções ambientais pode ser encontrado na laguna de Piratininga (Niterói, RJ; figura 1) descrito por Cunha \& Wasserman (2003) onde em 1995, foi construída uma comporta no canal por onde entrava água do mar na laguna, a qual tinha por objetivo palear o problema de contaminação por esgotos domésticos. A concepção do empreendimento era baseada em uma assertiva meramente hidráulica (“entrada, reservatório e saída”). Segundo a justificativa da época, a comporta permitiria o aumento do volume da laguna, diluindo assim a sua contaminação. O monitoramento da qualidade da água no ano que seguiu à instalação da comporta, concluiu que a barragem tinha causado redução do tempo de residência da água e, com isto, embora o volume 
de água da laguna estivesse maior, sua qualidade era ainda pior que antes da sua instalação. Quando a instalação da comporta festejava seu primeiro ano de existência, uma conjunção de fatores climáticos e oceanográficos provocou uma forte anoxia da laguna, provocando a mortandade de pelo menos 300 toneladas de peixe. As comunidades locais, que nunca foram totalmente favoráveis ao empreendimento, protestaram e ameaçaram dinamitar a comporta, diante do que, a Prefeitura decidiu inutilizar a sistema. Até hoje a estrutura da comporta permanece no local como um monumento à falta de visão interdisciplinar.

Mais recentemente, a Secretaria Estadual de Rios e Lagoas estabeleceu um Projeto de Recuperação do Sistema Lagunar de Piratininga-Itaipú (Secretaria Estadual de Rios $e$ Lagoas (SERLA), 2005) que consiste na construção de um túnel conectando o mar à laguna. Mais uma vez, a concepção do projeto resulta de uma visão unidiscplinar simplificada que está muito bem expressa no texto abaixo, retirado do documento que explica o projeto:

\section{... a fim de reverter a degradação ambiental predominante e propiciar o aumento do nível, altura e espelho d'água da Lagoa de Piratininga, favorecendo, assim, a renovação, circulação e melhoria da qualidade das águas}

Da mesma forma que em 1995, o problema é abordado segundo o conceito de "entrada, reservatório, saída”, onde o aumento do volume e a re-circulação da água levam à diluição dos esgotos.

Um problema que se coloca com freqüência nos procedimentos de recuperação de lagos e lagunas contaminadas por esgotos domésticos está relacionado à difusão do fósforo para a coluna d'água devido a um aumento da circulação da água no sistema (Friedrich et al., 2003; Linge \& Oldham, 2004). O fenômeno da difusão do fósforo na coluna d’água tem levado muitos programas de recuperação da qualidade da água de lagos e lagunas à decepção de ao final de um longo período não apresentar um aumento da transparência da água (Meijer et al., 1999). Em muitos casos, como em Piratininga, o estoque de fósforo do sedimento é limitado (Souza \& Wasserman, 1997) e com o passar dos anos este estoque se esgota e provavelmente a qualidade da água melhora. O problema é que não podemos prever quantos meses, ou anos, ou séculos este processo pode levar.

No caso de Piratininga existem soluções melhores, mais seguras e de longo prazo, mas que envolvem abordagens holísticas. Para encontrar tais soluções é preciso formar grupos interdisciplinares que tenham a capacidade de enxergar o problema segundo suas várias óticas.

\section{3- A MODELAGEM \\ FERRAMENTA HOLÍSTICA}

COMO

Um exemplo significativo da construção da abordagem holística tem aparecido na aplicação de modelos numéricos à solução e à previsão de impactos de atividades humanas no meio ambiente (Wasserman, 2000; Alves, 2003). Os modelos numéricos têm se mostrado muito eficientes e econômicos na determinação dos impactos, mesmo antes da construção das soluções (Koutitas, 1988; Rajar \& Cetina, 1997; Abreu et al., 1998; Gillibrand, 2001; Horiguchi et al., 2001; Suh, 2001; Balas \& Özhan, 2002). É um pouco da tentativa e erro referida na introdução deste artigo, mas sem os riscos de degradar o ambiente, já que todo o processo é simulado no computador. No caso de Piratininga, a determinação numérica do tempo de residência da água, o $\mathrm{T}_{50 \%}$ (tempo necessário para a troca de $50 \%$ da água da laguna pelos aportes das marés e das águas dos rios; Knoppers et al., 1991; Knoppers et al., 1999; Alves, 2003) poderia ter facilmente levado ao abandono do projeto, ou pelo menos ao seu ajuste. No caso uma melhor gestão da abertura e fechamento da comporta poderia amenizar os efeitos do acúmulo de água contaminada no sistema e até mesmo levar a uma melhoria da qualidade da água.

A modelagem hidráulica de cursos d'água, de lagoas e lagunas tem sido razoavelmente utilizada para se determinar soluções de gestão 
ambiental e de recursos hídricos. Lanna (2002) relata a importância da abordagem sistêmica na construção de modelos numéricos para a gestão de recursos hídricos. Em seu exemplo do rio dos Sinos (RS), ele demonstra como a representação simplificada de processos individualizados (por exemplo: as equações que descrevem a entrada e saída de água em um segmento do rio) permite a construção de um modelo que leva à simulação do todo. Embora o processo pareça cartesiano (reducionista), a ferramenta informática permite a integração dos processos de maneira iterativa tão complexa que não é concebível pelo raciocínio humano. É evidente, que a modelagem numérica no caso do exemplo do autor acima gera um resultado aproximado, já que as equações que simulam os processos são também aproximações. Neste tipo de modelagem determinística, quanto maior for 0 número de processos simulados, melhor será a sua representatividade.

Pode-se pensar que seria possível representar um número cada vez maior de processos, construindo modelos que simulassem com muita precisão o comportamento do meio. Contudo, as limitações para este processo, embora não estejam vinculadas à capacidade de cálculo dos computadores, recaem sobre o conhecimento preciso dos processos. Exemplificando, a vazão de cursos d’água é freqüentemente medida através de réguas limnimétricas que relacionam esta vazão à altura da água (Fill, 1987). Este modelo é construído assumindo que no trecho estudado a água tem uma viscosidade constante, e também o fundo tem uma declividade e rugosidade constantes. Contudo, em períodos de grande vazão é freqüente observarmos uma água muito mais carregada em finas partículas em suspensão, que podem atribuir um caráter mais viscoso à água, reduzindo assim a velocidade e a vazão, sem modificar a altura. Se o curso d’água é muito pequeno, esta variação será insignificante, contudo, para um rio como o
Amazonas, o erro pode ser da ordem de milhares de metros cúbicos.

Da mesma forma, pequenas imprecisões assumidas como condições de contorno do modelo podem se propagar ao longo das iterações dos cálculos e provocar resultados muito distintos dos observados. Um exemplo interessante resulta do trabalho de Knoppers et al. (1991), que construiu um modelo simplificado de tempo de residência em lagunas da Região dos Lagos (RJ). Neste modelo foram feitas aproximações que tinham por objetivo adaptar os processos à simplificada concepção da simulação. Assumiu-se assim, que em um sistema lagunar que tivesse várias sub-lagunas (sacos) interligadas considerar-se-ia que cada uma delas estaria diretamente ligada com o mar, e a altura (prisma, simbolizado pela letra P) da maré em cada um dos sacos seria reduzida concomitantemente com seu distanciamento do mar (indicado pela expressão $\mathrm{P} / \mathrm{N}$, onde $\mathrm{P}$ é a altura da maré e o divisor $\mathrm{N}$ é o fator de redução da altura). A Figura 2 mostra o exemplo da laguna de Saquarema. O modelo assumia ainda uma premissa de mistura completa, onde cada vez que a maré entrava nas lagunas, a água era completamente misturada e retirada do sistema na vazante.

Em trabalho posterior, Alves \& Wasserman (2002) construíram um modelo numérico unidimensional do sistema lagunar com o programa EcoS 3 (Gorley \& Harris, 1997; Harris \& Gorley, 1997), que utiliza equações governantes de dispersão e momentum pelo método das diferenças finitas. Diferentemente do trabalho de Knoppers et al. (1991), o modelo dos autores acima, considerava o sistema lagunar segundo um eixo unidimensional, onde a maré penetrava e atenuava-se até o saco mais interior, representando um sistema muito mais próximo da realidade. 


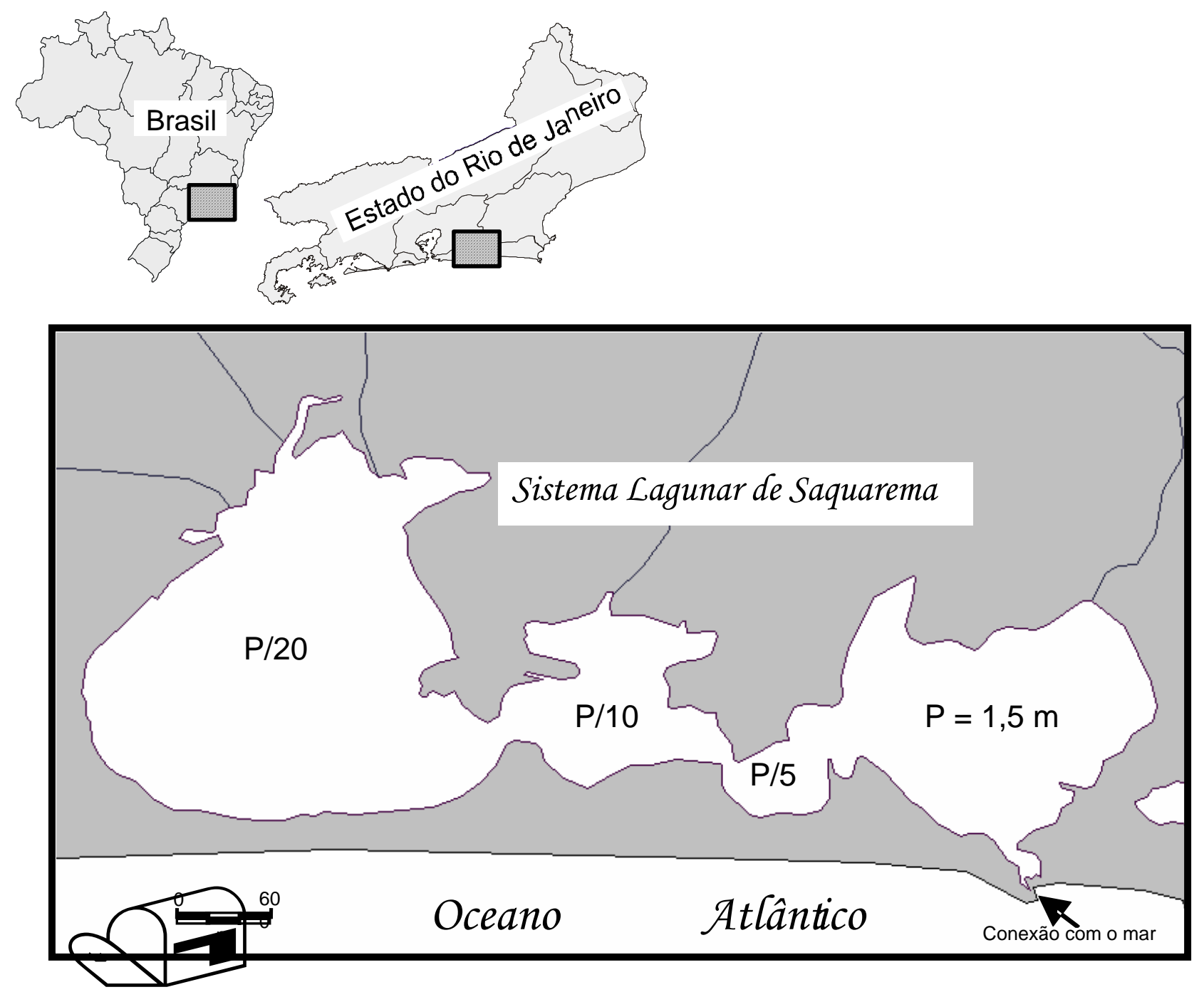

Figura 2: Esquema indicando como o prisma de maré (P) é atenuado segundo um fator $\mathrm{N}$ ao penetrar em cada uma das sub-lagunas. O Modelo de (Knoppers et al., 1991) assume que cada uma das lagunas está diretamente ligada ao mar e não ocorre nenhum tipo de interação entre elas.

No modelo numérico de diferenças finitas de Alves \& Wasserman (2002), graças à discretização do sistema, a água não mistura completamente, de maneira que uma gota d’água presente no saco mais interior leva muito mais tempo para sair do sistema. Além disto, a interação entre os diferentes sacos (em Knoppers et al., 1991, não há interação pois as lagunas estão ligadas diretamente com o mar) faz com que a gota d'água de um determinado ponto do sistema vá e volte com os ciclos de maré, levando muito mais tempo para atingir a saída do sistema lagunar.

As diferenças na determinação do tempo de residência aparecem representadas na tabela $1 \mathrm{e}$ têm uma implicação significativa em termos de capacidade de suporte do sistema ${ }^{4}$. Assim, o modelo unidimensional discretizado mostra que o sistema lagunar suporta uma carga de poluição

\footnotetext{
4 Capacidade de suporte é a quantidade de impacto ambiental que um sistema está apto a absorver sem deixar cair a sua qualidade.
} 
muito inferior àquela determinada pelo modelo anterior.

Tabela 1: Comparação dos tempos de renovação (em dias) segundo os modelos de (Knoppers et al., 1991) e de (Alves \& Wasserman, 2002).

\begin{tabular}{|l|c|c|}
\hline & $\begin{array}{c}\text { (Knoppers } \\
\text { et al., 1991) }\end{array}$ & $\begin{array}{c}\text { (Alves \& } \\
\text { Wasserman, } \\
\text { 2002) }\end{array}$ \\
\hline $\begin{array}{l}\text { Sub-laguna } \\
\text { conectada ao } \\
\text { mar (dias) }\end{array}$ & 8 & 24 \\
\hline $\begin{array}{l}\text { Sub-laguna } \\
\text { mais interna } \\
\text { (dias) }\end{array}$ & 14 & $>180$ \\
\hline
\end{tabular}

\section{4- CONCLUSÃO}

A natureza pode ser considerada como um sistema complexo e para que o homem entenda os processos que regem sua dinâmica é necessário abordá-la com uma visão multifocal. Embora alguns cientistas já tratem os problemas ambientais de maneira integrada, a ausência de métodos adequados à aplicação da abordagem holística dificulta sua disseminação.

Neste trabalho, foi demonstrado como a abordagem holística pode facilitar de maneira significativa 0 entendimento dos processos ambientais. O não entendimento de todos os fatores que afetam a dinâmica de um sistema complexo pode levar a conclusões errôneas. No caso da laguna de Piratininga, uma abordagem unidisciplinar, (hidráulica), levou à geração de uma solução muito simplista, que acabou se mostrando negativa para a saúde do ambiente

No caso da modelagem da laguna de Saquarema, após um período de quase 10 anos, foi possível identificar a magnitude do erro gerado por uma modelagem simplificada. Seria catastrófico se nos anos 90 tivéssemos dimensionado o potencial de crescimento populacional das margens do sistema lagunar para um valor até 10 vezes superior ao que viria a ser determinado no modelo mais recente.
A modelagem numérica tem evoluído muito e atualmente os exercícios de calibração e validação têm demonstrado a eficiência das previsões, contudo, com os modelos determinísticos a responsabilidade do modelador é muito grande. No caso de Saquarema, a ampliação e perenização do canal de conexão com o mar, causaram problemas que ultrapassaram a capacidade de previsão dos modelos. Em detrimento do projeto inicial e por falta de recursos, o quebra-mar de perenização foi construído muito curto, de tal forma que permitiu a entrada de ondas dentro da laguna, o que já algumas vezes provocou inundações nas margens. Embora a população esteja sofrendo bastante, e os pesquisadores envolvidos já tenham proposto soluções, a postura das autoridades ainda é de negligência. Todos estes aspectos, mesmo os pouco ambientais, fazem parte da abordagem holística.

\section{5- AGRADECIMENTOS}

Os autores agradecem os excelentes comentários feitos pelos dois relatores ad-hoc que contribuíram para uma melhoria significativa do conteúdo deste trabalho.

\section{6- REFERÊNCIAS BIBLIOGRÁFICAS}

Abreu, S. N., Pereira, M. E., \& Duarte, A. C. The use of a mathematical model to evaluate mercury accumulation in sediments and recovery time in a coastal lagoon (Ria de Aveiro, Portugal). Wat. Sci. Tech., v. 37, n. 6/7, p. 33-38, 1998.

Alves, A. R. Modelagem numérica como ferramenta de gestão ambiental. Aplicação ao sistema lagunar de Saquarema, RJ. Dissertação de Mestrado, Programa de Pós-Graduação em Ciência Ambiental, Universidade Federal Fluminense, Niterói. 2003. 97p.

Alves, A. R., \& Wasserman, J. C. Determinação do tempo de renovação em sistemas lagunares. Mundo Vida Alter. Est. Amb., v. 3, n. 1, p. 4853, 2002. 
Balas, L., \& Özhan, E. Three-dimensional modelling of stratified coastal waters. Est. Coastal Shelf Sci., v. 54, p. 75-87, 2002.

Cunha, L. C., \& Wasserman, J. C. Relationship between nutrients and macroalgal biomass in a Brazilian Coastal Lagoon: The impact of a lock construction. Chem. Ecol., v. 19, n. 4, p. 283298, 2003.

Fill, H. D. Informações Hidrológicas. In: F. T. Barth, C. T. Pompeu, H. D. Fill, C. E. M. Tucci, J. Kelman, \& B. P. F. Braga Jr. (Eds.), Modelos para o Gerenciamento de Recursos Hídricos, p. 94-210. São Paulo: Nobel/ABRH.1987.

Friedrich, J., Dinkel, C., Grieder, E., Radan, S., Secrieru, D., Steingruber, S., \& Wehrli, B. Nutrient uptake and benthic regeneration in Danube Delta lakes. Biogeochemistry, v. 64, n. 3, p. 373-398, 2003.

Gillibrand, P. A. Calculating Exchange times in a Scottish fjord using a two-dimensional, laterally-integrated numerical model. Est. Coastal Shelf Sci., v. 53, p. 437-449, 2001.

Gorley, R. N., \& Harris, J. R. W. ECoS3. Plymouth Marine Laboratory.1997. 36 p.

Harris, J. R. W., \& Gorley, R. N. An Introduction to Modelling Estuaries with ECoS3. Plymouth Marine Laboratory.1997. 45 p.

Horiguchi, F., Yamamoto, J., \& Nakata, K. A numerical simulation of the seasonal cycle of temperature, salinity and velocity fields in Tokyo Bay. Mar. Poll. Bull., v. 43, n. 7-12, p. 145-153, 2001.

Knoppers, B. A., Carmouze, J. P., \& MoreiraTurcq, P. F. Nutrient dynamics, metabolism and eutrophication of lagoons along the East Fluminense Coast, State of Rio de Janeiro. In: B. A. Knoppers, E. D. Bidone, \& J. J. Abrão (Eds.), Environmental Geochemistry of Coastal Lagoon Systems of Rio de Janeiro, Brazil, Vol. 6, p. 123-154. Niterói: UFF/FINEP.1999.

Knoppers, B. A., Kjerfve, B., \& Carmouze, J. P. Trophic state and water turn-over time in six choked coastal lagoons in Brazil. Biogeochemistry, v. 14, p. 149-166, 1991.

Koutitas, C. G. Mathematical Models in Coastal Engineering. London: Pentech Press.1988.

Lanna, A. E. Introdução. In: R. L. L. Porto (Ed.), Técnicas quantitativas para o gerenciamento de recursos hídricos, $2^{\mathrm{a}}$ ed., $\mathrm{p}$. 15-41. Porto Alegre: UFRGS/ABRH.2002.

Linge, K. L., \& Oldham, C. E. Control mechanisms for dissolved phosphorus and arsenic in a shallow lake. Appl. Geochem. , v. 19, n. 9, p. 1377-1389, 2004.

Meijer, M. L., de Boois, I., Scheffer, M., Portielje, R., \& Hosper, H. Biomanipulation in shallow lakes in The Netherlands: an evaluation of 18 case studies. Hydrobiol., v. 409, p. 13-30, 1999.

Rajar, R., \& Cetina, M. Hydrodynamic and water quality modelling: an experience. Ecol. Model., v. 101, p. 195-207, 1997.

Secretaria Estadual de Rios e Lagoas (SERLA). Recuperação do Sistema Lagunar de Piratininga Itaipu, http://www.serla.rj.gov.br/serlagoa_piratininga. asp 2005.

Souza, N. M., \& Wasserman, J. C. The fate of anionic surfactants in a tropical choked lagoon in SE Brazil. Ci. Cult. J. Braz. Ass. Adv. Sci, v. 49, n. 1/2, p. 130-135, 1997.

Suh, S. W. A hybrid near-field/far-field thermal discharge model for coastal areas. Mar. Poll. Bull., v. 43, n. 7-12, p. 225-233, 2001.

Vallega, A. The role of integrated scientific approach facing the changing ocean policy. The case of the Mediterranean. Prog. Oceanogr., v. 44, n. 1-3, p. 411-431, 1999.

Wasserman, J. C. Estudo do Impacto Ambiental da Abertura da Barra da Laguna de Saquarema. Prefeitura Municipal de saquarema. Saquarema. 2000. 